\title{
2-517-4
}

\section{Fibulin-1 integrates subendothelial extracellular matrices and contributes to anatomical closure of the ductus arteriosus.}

\author{
$\underline{\text { Satoko }}$ Ito $^{1,2}$, Utako Yokoyama ${ }^{1,2}$, Taichi Nakakoji ${ }^{2}$, Yuko Kato ${ }^{1}$, Junichi Saito ${ }^{1,2}$, Naoki Nicho ${ }^{2}$, \\ Masuda Munetaka ${ }^{3}$, Toshihide Asou ${ }^{4}$, Yoshihiro Ishikawa ${ }^{2}$
}

${ }^{I}$ Department of Physiology, Tokyo Medical University, ${ }^{2}$ Cardiovascular Research Institute, Yokohama City University, ${ }^{3}$ Department of Surgery, Yokohama City University, ${ }^{4}$ Department of Cardiovascular Surgery, Kanagawa Children's Medical Center

Objective: COX inhibitors targeting smooth muscle cell (SMC) contraction represent the only pharmacological treatment for patent ductus arteriosus (PDA), but $>30 \%$ patients are resistant to the current therapies. Intimal thickening (IT), occurs in the subendothelial region of DA to bring anatomical DA closure. We investigated the role of fibulin-1 in DA anatomical closure to seek a new IT-inducing pharmacological therapy.

Approaches and results: Microarray analysis demonstrated that fibulin-1 was the most up-regulated gene by stimulation of EP4 in DA-SMCs. EP4-induced fibulin-1 expression was mediated through the phospholipase Cprotein kinase C-noncanonical nuclear factor-kappa B pathway. We performed FACS analysis and found that fibulin -1 binding protein versican was derived from DA-endothelial cells. Immunofluorescence demonstrated that fibulin-1 and versican V0/V1 were co-expressed at the IT of wild-type DA. In the DA of EP4-deficient mouse (Ptger4 $4^{-}$), fibulin-1was largely attenuated and showed PDA. All of fibulin-1-deficient mice exhibited PDA with hypoplastic IT, and fibulin-1 protein administration restored IT formation of Ptger 4. Furthermore, $30 \%$ of versican deleted mice lacking a hyaluronan binding site displayed PDA.

Conclusions: Fibulin-1 contributes to DA closure by forming an environment favoring directional SMC migration toward the subendothelial region in combination with versican and hyaluronan. Targeting fibulin-1 upregulation may provide the basis for therapeutic strategies for inducing anatomical DA closure. 